\title{
ARTICLE OPEN Dichotomy in ultrafast atomic dynamics as direct evidence of polaron formation in manganites
}

\author{
Junjie $\mathrm{Li}^{1}$, Wei-Guo Yin ${ }^{1}$, Lijun Wu ${ }^{1}$, Pengfei Zhu ${ }^{1,2}$, Tatianna Konstantinova ${ }^{1,3}$, Jing Tao ${ }^{1}$, Junjie Yang ${ }^{4}$, Sang-Wook Cheong ${ }^{4,5}$,
} Fabrizio Carbone ${ }^{6}$, James A Misewich ${ }^{1,3}$, John P Hill ${ }^{1}$, Xijie Wang ${ }^{1,7}$, Robert J Cava ${ }^{8}$ and Yimei Zhu ${ }^{1,3}$

Polaron transport, in which electron motion is strongly coupled to the underlying lattice deformation or phonons, is crucial for understanding electrical and optical conductivities in many solids. However, little is known experimentally about the dynamics of individual phonon modes during polaron motion. It remains elusive whether polarons have a key role in materials with strong electronic correlations. Here we report the use of a new experimental technique, ultrafast MeV-electron diffraction, to quantify the dynamics of both electronic and atomic motions in the correlated $\mathrm{LaSr}_{2} \mathrm{Mn}_{2} \mathrm{O}_{7}$. Using photoexcitation to set the electronic system in motion, we find that Jahn-Teller-like $\mathrm{O}, \mathrm{Mn}^{4+}$ and $\mathrm{La} / \mathrm{Sr}$ displacements dominate the lattice response and exhibit a dichotomy in behaviour-overshoot-and-recovery for one sublattice versus normal behaviour for the other. This dichotomy, attributed to slow electronic relaxation, proves that polaron transport is a key process in doped manganites. Our technique promises to be applicable for specifying the nature of electron-phonon coupling in complex materials.

npj Quantum Materials (2016) 1, 16026; doi:10.1038/npjquantmats.2016.26; published online 25 November 2016

\section{INTRODUCTION}

The temporal correlation between electron motion and the atomic lattice distortion ${ }^{1-7}$ is considered essential to electronic transport, in behaviour ranging from resistance-free flow ${ }^{2}$ to selftrapping, ${ }^{3}$ however, obtaining direct experimental information about the dynamics of electron-lattice coupling on the picosecond timescale is a daunting problem. As atomic and electronic masses differ by 3-5 orders of magnitude, it has been generally assumed (the Born-Oppenheimer approximation) that the motions of atomic nuclei and electrons can be considered as separate, with the electrons in their eigenstates for any given positions of the underlying atomic lattice. In condensed matter systems with strong electron-electron or electron-lattice interactions, ${ }^{7,8}$ however, the effective masses of the conduction electrons can be significantly increased. In other words, the electrons are slowed down and the atoms in the lattice can adjust their positions in a timely manner in response to changes in the electronic states, resulting in a strongly coupled system or 'polarons'. ${ }^{1}$ Recently, the use of ultrafast pump-probe techniques to drive electronic systems out of equilibrium has emerged as a powerful means for altering electronic states, e.g., to yield photoinduced transient superconductivity in cuprates s, $^{4,10}$ and an antiferromagnetic to ferromagnetic transition in manganites. ${ }^{11}$ This opens the possibility of observing the correlation of the electronic and atomic motions that occurs on entry into a nonequilibrium state. $^{10}$ Here we pursue this idea through the quantitative ultrafast and ultrahigh-energy electron diffraction (MeV-UED) characterisation of the prototypical half-doped bi-layer manganite, $\mathrm{LaSr}_{2} \mathrm{Mn}_{2} \mathrm{O}_{7}$. We are able to quantitatively follow, on the picosecond timescale, the changes in the crystal structure and electronic system of this material in response to photoexcitation, and from those observations find an unanticipated correlation of the two systems that illustrates the power of this new technique in understanding the electronic properties of condensed matter systems.

$\mathrm{LaSr}_{2} \mathrm{Mn}_{2} \mathrm{O}_{7}$ belongs to the family of $A$-site-doped layered manganites $A_{n+1} \mathrm{Mn}_{n} \mathrm{O}_{3 n+1}$ (where $A$ is a rare-earth element and $n=2)$. Below its charge and orbital ordering (OO) temperature $T_{\mathrm{CO}-\mathrm{OO}}=210 \mathrm{~K}$, the two types of $\mathrm{Mn}$ ions order in the double $\mathrm{MnO}_{2}$ layers in a checkerboard pattern of nominal $\mathrm{Mn}^{3+}$ and $\mathrm{Mn}^{4+}$ (Figure 1a), yielding a 'charge ordered' (CO) state. ${ }^{12}$ At the same temperature, the excess electron present on the $\mathrm{Mn}^{3+}$ undergoes an ordering transition such that it occupies, alternatingly, the $3 x^{2}-r^{2}$ and $3 y^{2}-r^{2}$ orbitals on adjacent atoms along the crystallographic $b$ direction, ${ }^{13,14}$ a phenomenon known as OO. The coexisting $\mathrm{CO}-\mathrm{OO}$ state in the half-doped manganites has been extensively studied because it can yield insight into the colossal magnetoresistance effect observed in perovskite-related manganites. As the $\mathrm{CO}$ is coupled to breathing-mode lattice distortions and the $\mathrm{OO}$ is coupled to Jahn-Taller-mode lattice distortions, ${ }^{15-17}$ it has been suggested that the electrons in doped manganites are heavily dressed with these lattice distortions, forming polarons, and that the formation, melting and ordering of the polarons are the key to the colossal magnetoresistance effect. ${ }^{3,18-22}$ Thus, this system is ideal for studying polarons, with the accompanying dynamics of the interplay of electronic and lattice degrees of freedom in a complex solid.

The $\mathrm{OO}$ and $\mathrm{CO}$ in the manganites are directly measurable as superlattice peaks in X-ray and electron diffraction. To discover the

\footnotetext{
${ }^{1}$ Division of Condensed Matter Physics and Materials Science, Brookhaven National Laboratory, Upton, NY, USA; ${ }^{2}$ Department of Physics and Astronomy, Shanghai Jiao Tong University, Shanghai, China; ${ }^{3}$ Department of Physics and Astronomy, Stony Brook University, Stony Brook, NY, USA; ${ }^{4}$ Laboratory of Pohang Emergent Materials, Pohang University

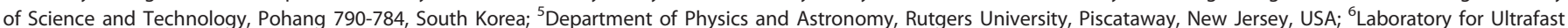
Microscopy and Electron Scattering, ICMP, Ecole Polytechnique Fédérale de Lausanne, CH-1015 Lausanne, Switzerland; ${ }^{7}$ SLAC National Accelerator Laboratory, Menlo Park, CA, USA and ${ }^{8}$ Department of Chemistry, Princeton University, Princeton, NJ, USA.
}

Correspondence: Y Zhu (zhu@bnl.gov)

Received 23 September 2016; revised 14 October 2016; accepted 18 October 2016 
a
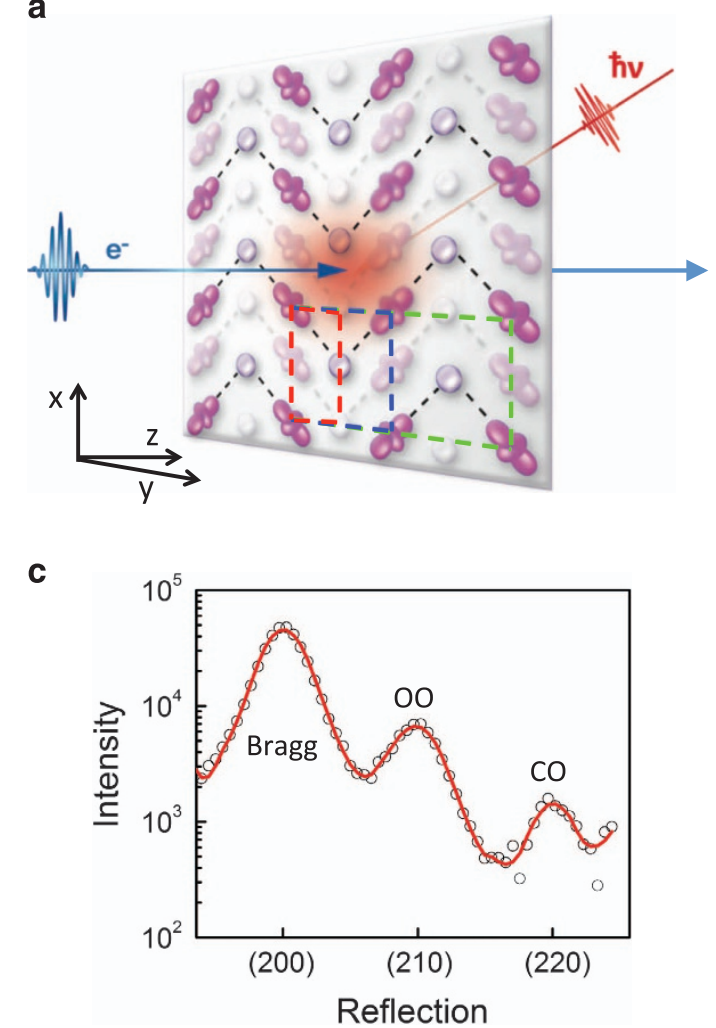

b

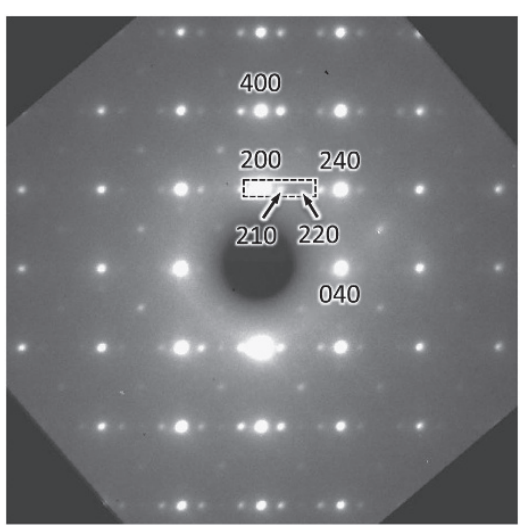

d

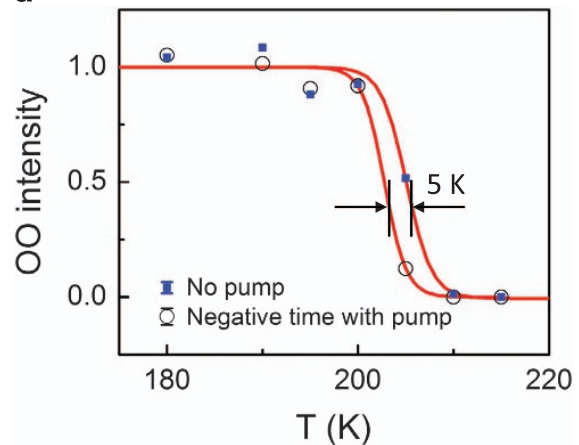

Figure 1. Experimental geometry and static characterisation of orbital ordering. (a) Geometrical arrangement of pump and probe at the sample position in the MeV-UED experiment. An $800 \mathrm{~nm}$ laser was used to pump the $\mathrm{LaSr}_{2} \mathrm{Mn}_{2} \mathrm{O}_{7}$ single crystal that exhibits CO (double unitcell in blue) and $\mathrm{OO}$ (quadruple unit-cell in green). (b) Typical electron diffraction pattern at $77 \mathrm{~K}$ recorded before time 0 with an accumulation of 800 shots. The bright spots marked as ( $h k 0)$ with $k=4 n, k=4 n \pm 1$ and $k=4 n \pm 2$ correspond to the Bragg, OO and CO reflection peaks, respectively. (c) Integrated intensity profile of the (200) Bragg peak, the (210) OO and (220) CO superlattice peaks in the boxed area in (b), fitted with a combined Lorentzian and Gaussian function. (d) Static measurement of temperature dependence of the OO across the phase transition temperature $210 \mathrm{~K}$. The blue solid squares were recorded without using the laser pump and the black open circles were recorded with the laser pump (of the $4 \mathrm{~mJ} / \mathrm{cm}^{2}$ fluence and the $10 \mathrm{~Hz}$ repetition rate) turned on after the probing electron pulse. The red lines are guide to the eyes, indicating a residue heating of no more than $5 \mathrm{~K}$ during the pump-probe experiment.

dynamics of atom-specific lattice distortions during polaron formation through time-dependent structural refinement, simultaneous retrieval of all relevant reflections in a single diffraction pattern is highly desirable, but challenging. Recent ultrafast X-ray diffraction measurements revealed that photoexcitation suppressed the $\mathrm{CO}$ and $\mathrm{OO}$ in half-doped manganites $\mathrm{La}_{0.5} \mathrm{Sr}_{1.5} \mathrm{MnO}_{4}$ $(n=1$; refs 23,24$)$ and $\operatorname{Pr}_{0.5} \mathrm{Ca}_{0.5} \mathrm{MnO}_{3}(n=\infty$; ref. 25$)$. However, these experiments involved rotation of the samples and changes in the incident X-ray energy and flux in order for different classes of peaks to be measured, making it not only hard to precisely clock the 'time-zero', but, more importantly, also difficult to properly renormalise the intensities of those separately measured reflections. In contrast, the UED technique typically using tens-ofkeV electrons can simultaneously measure $\mathrm{CO}$ and $\mathrm{OO}$ superlattice peaks, but the number of reflections is usually limited for structural refinement. ${ }^{26-29}$ It was recently demonstrated ${ }^{30,31}$ that 2.8 MeV electrons can double the number of accessible reflections than $50 \mathrm{keV}$ electrons. ${ }^{26}$ Such an expansion of high-order reflections, which are very sensitive to atomic displacement, is crucial to the accurate detection of phonons. Here, we use the recently commissioned accelerator-based relativistic MeV-UED system that is achievable of $130 \mathrm{fs}$ temporal resolution $\mathrm{n}^{30,31}$ to directly measure the dynamic paths of atoms during the suppression of the $\mathrm{OO}$ and $\mathrm{CO}$ states in the $\mathrm{LaSr}_{2} \mathrm{Mn}_{2} \mathrm{O}_{7}$ single crystal under $800 \mathrm{~nm}(1.55 \mathrm{eV})$ laser excitation. The pump-probe set-up is illustrated in Figure $1 \mathrm{a}$ and described in Methods, along with additional advantages of MeV-UED. Eighty-six reflections with many associated to the $\mathrm{OO}$ and $\mathrm{CO}$ in the (001) zone are recorded simultaneously, enabling us to extract previously undisclosed atomic dynamics that strongly complements the earlier studies of photoinduced phase transitions in manganites.

\section{RESULTS}

A typical (001) diffraction pattern of $\mathrm{LaSr}_{2} \mathrm{Mn}_{2} \mathrm{O}_{7}$ at $77 \mathrm{~K}$ is shown in Figure 1b. The appearance of a series of sharp satellite spots indicates the existence of the superstructure modulation (Figure 1c). The superlattice spots ( $h k 0)$ with $k=4 n \pm 1$ and $k=4 n \pm 2$ represent the $\mathrm{OO}$ and the $\mathrm{CO}$, respectively. The notation is based on space group Bbmm with the lattice constants $a=0.5443 \mathrm{~nm}, b=1.0194 \mathrm{~nm}$ and $c=1.9816 \mathrm{~nm}$. The reflections (210) and (220) correspond, respectively, to $(1 / 4,1 / 4,0)$ and $(1 / 2,1 / 2,0)$ defined in the one-Mn Brillouin zone convention. ${ }^{13}$

\section{Photoinduced suppression of the $\mathrm{CO}$ and $\mathrm{OO}$}

Figure $2 \mathrm{a}$ and $\mathrm{b}$ shows the evolution of the peak intensity $I(t)$ of the $\mathrm{CO}$ and $\mathrm{OO}$ superlattice reflections as a function of time at $77 \mathrm{~K}$, normalised by the intensities before time zero $\left(I_{0}\right)$ at pump fluence of $4 \mathrm{~mJ} / \mathrm{cm}^{2}$. Both the $\mathrm{CO}$ and $\mathrm{OO}$ intensities drop quickly upon photoexcitation, indicating a reduction of both charge and orbital order parameters. After $5 \mathrm{ps}$, the $\mathrm{OO}$ intensities decrease by 

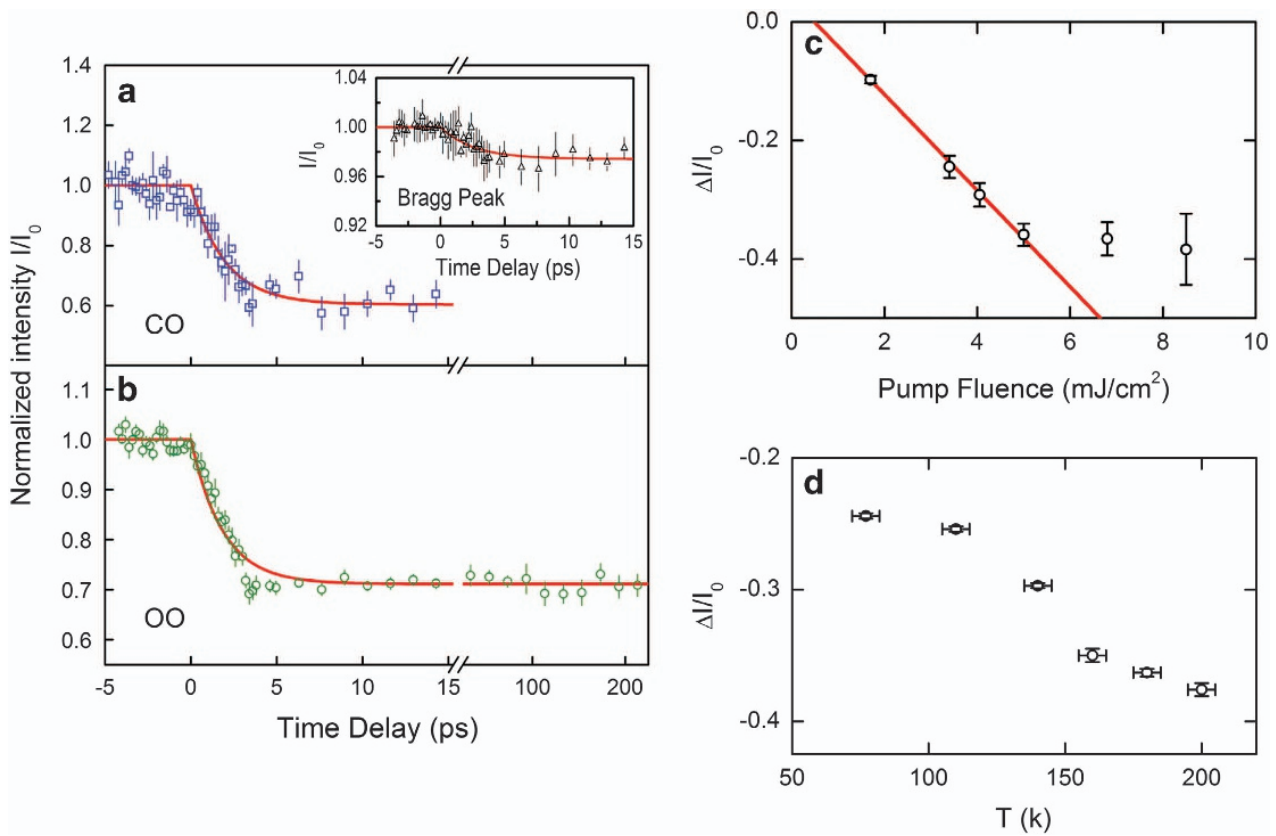

Figure 2. Temporal evolution of the lattice, charge and orbital ordering. (a) The $\mathrm{CO}$ and Bragg peak intensities (inset); (b) the OO peak intensity for the pump fluence of $4 \mathrm{~mJ} / \mathrm{cm}^{2}$ and the base sample temperature of $77 \mathrm{~K}$. The intensities, normalised by those before photo-excitation, are averaged over 4 high-intensity CO peaks of the $\{220\}$ family, 8 Bragg peaks of the $\{400\},\{600\},\{040\}$ and $\{080\}$ family, and 12 high-intensity 00 peaks of the $\{210\},\{410\}$ and $\{610\}$ family, respectively. The red lines are the fitting curves of an exponential function with time constants $\tau=1.86 \pm 0.12,1.97 \pm 0.30$ and $2.59 \pm 0.45 \mathrm{ps}$ for the $\mathrm{OO}, \mathrm{CO}$ and Bragg peaks, respectively. (c) The pump-fluence dependence of the change in the $\mathrm{OO}$ peak intensity at $77 \mathrm{~K}$. Below the saturation fluence, $\sim 5 \mathrm{~mJ} / \mathrm{cm}^{2}$, the amplitude change shows a linear dependence on the fluence, and the intercept with $\Delta l / I_{0}=0$ at $0.5 \mathrm{~mJ} / \mathrm{cm}^{2}$ represents a minimum fluence requirement for the photoinduced suppression of OO. (d) The basesample-temperature dependence of the changes in the $0 O$ peak intensity upon the $3.4 \mathrm{~mJ} / \mathrm{cm}^{2}$ laser pumping.

$\sim 28 \%$ over the maximum time delay studied of 200 ps. Similar behaviour was observed for the CO peaks. By fitting the curves to an exponential decay, the time constants associated with the $\mathrm{CO}$ and $\mathrm{OO}$ phase transition dynamics were derived, being $\tau_{\mathrm{CO}}=1.97 \pm 0.30 \mathrm{ps}$ for $\mathrm{CO}$ and $\tau_{\mathrm{OO}}=1.86 \pm 0.12 \mathrm{ps}$ for OO, identical within the measurement errors. In contrast, the decay of the Bragg peak intensity (the inset of Figure 2a), representing the average structure, exhibits a time constant of $\tau_{\text {Bragg }}=2.59 \pm 0.45 \mathrm{ps}$ with a much weaker, $\sim 2.5 \%$, reduction. Figure $2 \mathrm{c}$ presents relative reduction of the $\mathrm{OO}$ superlattice peak as a function of pump fluence up to $10 \mathrm{~mJ} / \mathrm{cm}^{2}$. It clearly displays a saturation point at $\sim 5 \mathrm{~mJ} / \mathrm{cm}^{2}$ : the OO intensity linearly decreases as the pump fluence increases from zero to $5 \mathrm{~mJ} / \mathrm{cm}^{2}$ and then remains unchanged at $-37 \%$ for higher fluences. Note that qualitatively similar results were observed in ultrafast $\mathrm{X}$-ray diffraction experiments on other half-doped $A_{n+1} \mathrm{Mn}_{n} \mathrm{O}_{3 n+1}$ manganites. ${ }^{23,25}$ Yet, the dynamics appears to be much faster in $\operatorname{Pr}_{0.5} \mathrm{Ca}_{0.5} \mathrm{MnO}_{3}$ $\left(n=\infty ; \tau_{\mathrm{OO}} \sim 0.5 \mathrm{ps}\right.$ in ref. 25$)$ with complete suppression of $\mathrm{OO}$ at the fluence of $5 \mathrm{~mJ} / \mathrm{cm}^{2}$. The slow dynamics in $\mathrm{LaSr}_{2} \mathrm{Mn}_{2} \mathrm{O}_{7}$ sets a clearer stage that the atomic motion may be cooperative, driven non-thermally through the rearrangement of charges.

The much weaker photoinduced suppression of the Bragg peak intensity suggests that the mechanism of an increased DebyeWaller (DW) factor, i.e., increased disorder in the superlattice due to laser heating, is unlikely to account for the observed large suppression of the CO/OO peak intensities. This is because the increase of DW would basically cause the same percentage drop of the intensities for both Bragg peak and its satellite CO/OO peaks. To have a proper understanding of the impact of laser heating, we performed the MeV-UED experiments at different base sample temperatures for the $3.4 \mathrm{~mJ} / \mathrm{cm}^{2}$ fluence. As shown in Figure $2 \mathrm{~d}$, the $\mathrm{OO}$ remains substantial at $200 \mathrm{~K}$; however, it would have disappeared if the sample was heated to $T_{\mathrm{CO}-\mathrm{OO}}=210 \mathrm{~K}$. Hence, the overall heating of the sample must be less than $10 \mathrm{~K}$. If all absorbed laser energies convert to thermal energy, the temperature increased by a single pulse is estimated to be less than $90 \mathrm{~K}$ (Supplementary Note 2). However, the absorbed energy also dissipates, resulting in a stable temperature distribution across the sample and a much smaller increase in temperatureat negative time delay with respect to the next pump-probe measurement. The residue heating in our set-up was measured to be $\sim 5 \mathrm{~K}$ (Figure 1d), which agrees with the calculated upper bound temperature of $12 \mathrm{~K}$ (Supplementary Note 2 and Supplementary Figure S4). Therefore, the sample temperature is estimated to be lower than $87 \mathrm{~K}$ during the measurements. In comparison, to thermally induce the observed large suppression of the $\mathrm{OO}$ peak intensity, the sample needs to be heated to above $160 \mathrm{~K}^{32}$ Hence, we conclude that the observed 00 dynamics is due to photoinduced electronic excitation.

Structural evolution and the associated phonon modes

We proceed to present our most significant findings of this work achieved by quantitatively analysing the simultaneously observed Bragg, $\mathrm{CO}$ and $\mathrm{OO}$ reflections in the (001) pattern in a series of time delays. The key features of the crystal structure are illustrated in Figure $3 \mathrm{a}$ and $\mathrm{b}$. The oxygen octahedra surrounding the $\mathrm{Mn}^{3+}$ sites are subjected to the Jahn-Teller distortion that yields the elongated $\mathrm{Mn}-\mathrm{O}$ bonds along the $\cdots-\mathrm{Mn}^{3+}-\mathrm{Mn}^{4+}-\mathrm{Mn}^{3+}-$ $\mathrm{Mn}^{4+}-\ldots$ zigzag chain and then the shortened $\mathrm{Mn}-\mathrm{O}$ bonds in the other directions. ${ }^{33}$ Importantly, such oxygen octahedral distortion is accompanied by the deviation of both $\mathrm{Mn}^{4+}$ and $\mathrm{La} / \mathrm{Sr}$ (A-site cation) from their high-symmetry (space group $14 / \mathrm{mmm}$ ) positions; thus, the Jahn-Teller phonon mode consists of the collective displacements of $\mathrm{O}, \mathrm{Mn}^{4+}$ and $\mathrm{La} / \mathrm{Sr}$ atoms. Meanwhile, the oxygen octahedra surrounding the $\mathrm{Mn}^{4+}$ sites are subjected to the breathing-mode distortion with reduced volume. On the basis of these major lattice distortion modes (oxygen and $\mathrm{Mn}^{4+}$ 
a

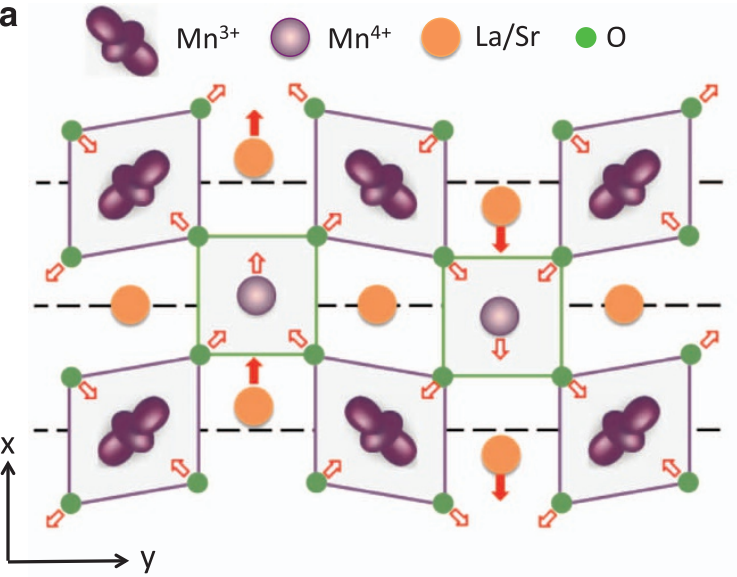

C

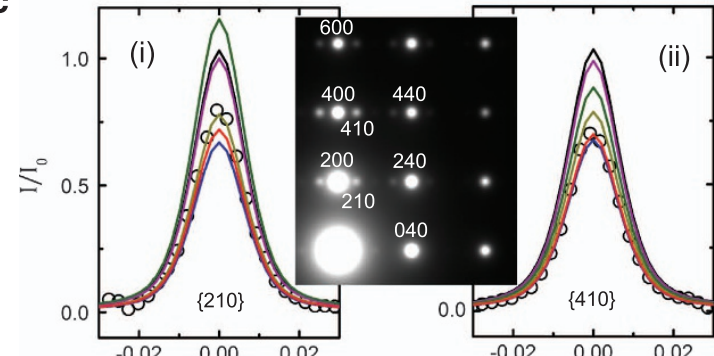

b

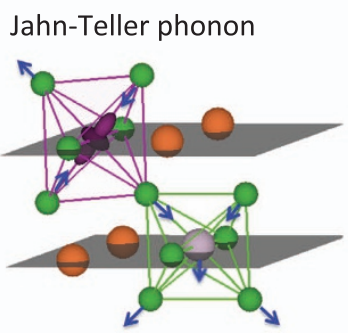

Rotation phonon

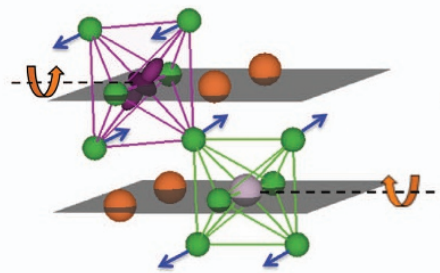

La/Sr phonon

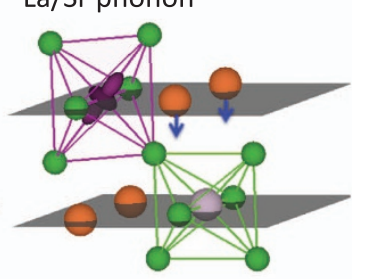

Breathing phonon

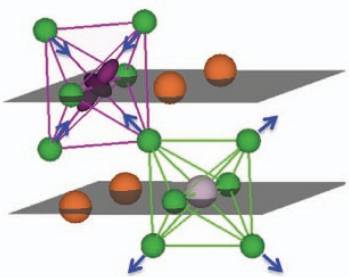

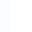
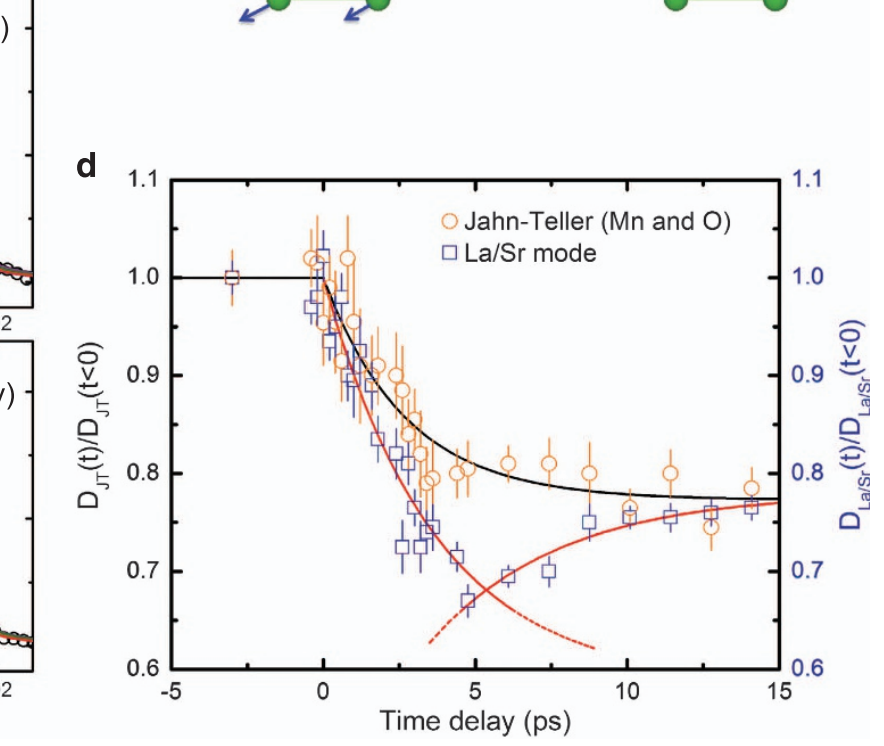

Figure 3. Separating various phonon modes. (a) Schematic view of the Jahn-Teller distortion related to $\mathrm{O}$ atom movement (open red arrows) accompanied by the cooperative displacement of $\mathrm{La} / \mathrm{Sr}$ and $\mathrm{Mn}^{4+}$ (solid and open red arrows) in $\mathrm{LaSr}_{2} \mathrm{Mn}_{2} \mathrm{O}_{7}$. (b) Four typical phonon modes. (c) Separating the roles of various phonon modes by quantitative analysis of the intensity reduction of 00 peak (inset) (i) $\{210\}$, (ii) $\{410\}$, (iii) $\{610\}$ and (iv) $\{430\}$ in quasi-equilibrium (14 ps after photoexcitation). The peak intensities are normalised to their respective values before time zero. A combined phonon mode of Jahn-Teller-like $\left(\mathrm{O}, \mathrm{Mn}^{4+}\right)$ and $\mathrm{La} / \mathrm{Sr}$ displacements (red lines) shows the best agreement with experiment with $X^{2}=0.003$ (see Supplementary Table S2). (d) Distinctive time dependences of the Jahn-Teller-like $\left(\mathrm{O}, \mathrm{Mn}^{4+}\right)$ and La/Sr displacements. The former (black circles) exhibits a single exponential decay with a time constant of $2.72 \mathrm{ps}$, while the latter (blue squares) possesses a two-step behaviour, namely, a 3.62 ps decay followed by a 4.32 ps recovery. The displacements are normalised to their respective values before time 0 . The error bars were obtained from the normalised JT and La/Sr displacement that yields 1 standard deviation $\pm \sigma$ from the mean value of the OO peak intensities shown in Figure $2 b$.

Jahn-Teller, breathing, rotation and $\mathrm{La} / \mathrm{Sr}$ ), we calculated the diffraction patterns and compared them with the observations (see Methods). We found that, despite the clear changes in the $\mathrm{OO}$ and $\mathrm{CO}$ reflection intensities, the crystal symmetry of the system remains (space group Bbmm). Furthermore, we identified that the 3200 superlattice peaks dominate the fitting because of the generally much weaker $\mathrm{CO}$ peak intensities (Figure $1 \mathrm{~b}$ ) and the little change in the Bragg peak intensities (Figure 2a). Below we first show in Figure $3 c$ our analysis of the atom-specific lattice distortions at one specific time, $t=14$ ps after the photoexcitation at which the system is in quasiequilibrium. Then, we present the results for the series of time delays in Figure $3 \mathrm{~d}$.

Figure $3 c$ shows the comparison of the intensities of the observed $\{210\},\{410\},\{610\}$ and $\{430\}$ OO superlattice reflections between the experiments (open circles) and the simulations for the aforementioned various lattice distortion modes (coloured lines), both individually and combinationally at quasi-equilibrium of $14 \mathrm{ps}$. To reduce the number of free parameters, the related atoms were grouped together and displaced proportionally based on the crystal symmetry. The atomic positions before time zero were used as reference, which yields unity in diffraction intensity (normalised intensity) for all the reflections. The standard $x^{2}$ criterion was used to assess the goodness of fit and the $x^{2}$ values are listed in Supplementary Table S2 (see Supplementary Note 1). The results indicate that none of the individual lattice distortion modes can match well with the observed intensity changes in the experiment. Specifically, the breathing and rotation modes of the oxygen octahedra have negligible impact on the quality of the fit. By contrast, the impact of the Jahn-Teller-associated $\mathrm{O}, \mathrm{Mn}^{4+}$ and $\mathrm{La} / \mathrm{Sr}$ displacements are all significant, in agreement with their 
strong coupling illustrated in Figure 3a). An excellent fit (with $x^{2}$ almost one order of magnitude smaller than for any individual mode) is found to result from cooperative Jahn-Teller-like ( $\mathrm{O}$ and $\mathrm{Mn}^{4+}$ ) and $\mathrm{La} / \mathrm{Sr}$ lattice distortions, with similar suppressions of $\sim 23 \%$ from their original ones, indicating the intimate relationship between the Jahn-Teller lattice distortion and the OO. The refined atomic positions before and after the photoexcitation are listed in Supplementary Table S1.

The result that the best fit comes from cooperative Jahn-Tellerlike $\left(\mathrm{O}, \mathrm{Mn}^{4+}\right)$ and $\mathrm{La} / \mathrm{Sr}$ displacements holds for all time delays (see Supplementary Figure S3). Any other combination of two individual modes yields worse results, whereas three and four combinations show little improvement of the goodness of the fit (see caption of Supplementary Figure S3b) for 10-14 ps time delays we tested. Therefore, we conclude that the Jahn-Teller-like $\mathrm{O}, \mathrm{Mn}^{4+}$ and $\mathrm{La} / \mathrm{Sr}$ displacements dominate the lattice response to the photoexcitation. Figure $3 \mathrm{~d}$ shows the time dependence of these lattice distortions. It is remarkable that these Jahn-Tellermode-related lattice distortions have different dynamic behaviours upon photoexcitation, as can be seen from the evolution of the atomic displacements in time. The $\mathrm{O}$ and $\mathrm{Mn}^{4+}$ lattice distortions show a single exponential drop with a time constant of $2.72 \mathrm{ps}$. By contrast, the La/Sr lattice distortion decay is completely different, described by a two-step behaviour, namely first a 3.62 ps exponential decay followed by a 4.32 ps recovery. In other words, the $\mathrm{La} / \mathrm{Sr}$ lattice distortion overshoots during the first $5 \mathrm{ps}$, before changing course to result in all the distortions converging to reach a quasiequilibrium state at $14 \mathrm{ps}$. This dichotomy of overshoot and recovery versus normal dynamics is robust, but hidden in the averaged $\mathrm{OO}$ peak intensity (Figure $2 \mathrm{~b}$ ). It becomes visible only after the more rigorous analysis of the large data set accessible with the MeV-UED method.

Our dynamical structural refinement can also help to separate the Debye-Waller effect. The atomic displacement corresponding to the observed $28 \%$ decrease of $\mathrm{OO}$ peak intensity yields a $1 \%$ increase in the Bragg peak intensity non-thermally (unrelated to the DW factor). As we experimentally observed a $2.5 \%$ drop of the Bragg peaks at $14 \mathrm{ps}$, the change of the DW factor is likely to contribute a $3.5 \%$ intensity drop of the Bragg peaks. Thus, we attribute the decay of Bragg intensities mostly to the lattice heating, and the suppression of the $\mathrm{OO}$ reflections is driven nonthermally through the rearrangement of charges.

\section{DISCUSSION}

A likely scenario for the overshoot-recovery versus normal dichotomy observed is the unequal involvement of the electron dynamics in determining the behaviour of different parts of the atomic system during the first 5 ps. To consider this in more detail, first it is necessary to determine what microscopic electronic process is induced by the $1.55 \mathrm{eV}$ photons. The Jahn-Teller distortion is known to split the twofold degenerate $e_{\mathrm{g}}$ levels on

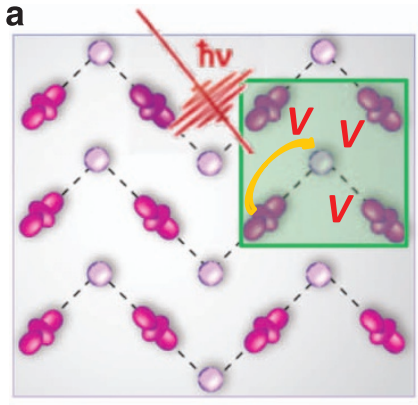

d $\mathrm{z}^{2}-\mathrm{z}^{2}$ transition

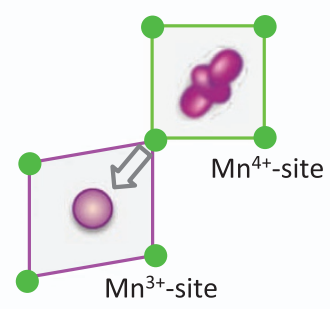

b intrasite transition

C $\Delta E=2 E_{\mathrm{JT}}$

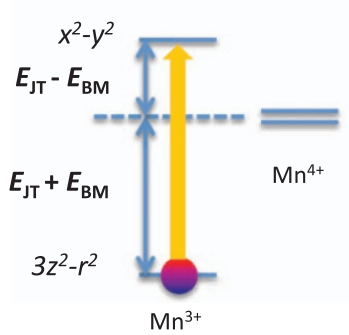

intersite transition

$$
\Delta E=E_{\mathrm{JT}}+E_{\mathrm{BM}}+3 V
$$

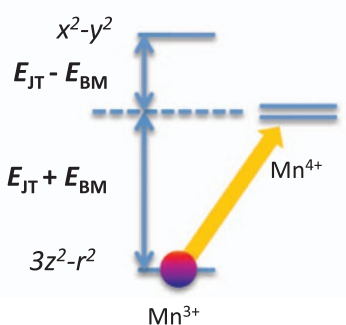

e
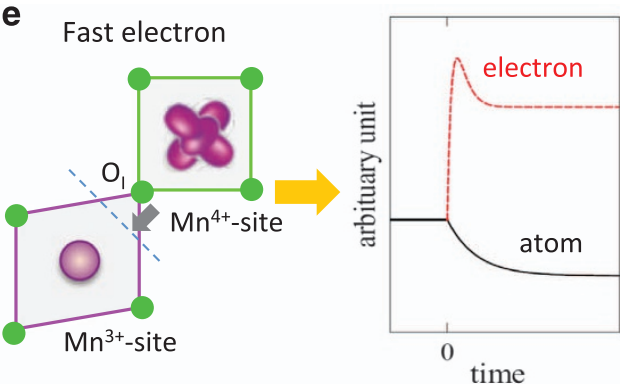

f
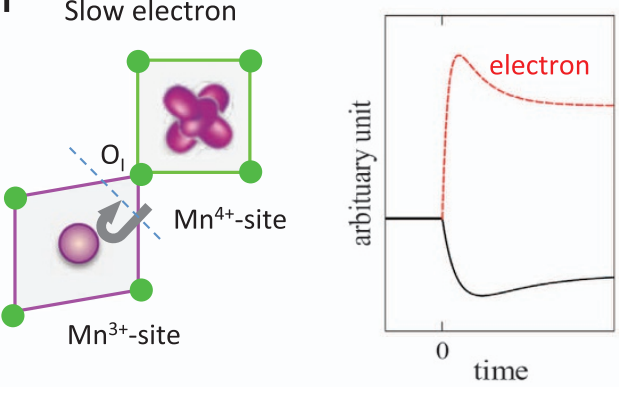

Figure 4. Schematics of the photoinduced electronic transition. (a) Schematic view of the orbital ordering. The yellow arrow indicates that an $\mathrm{e}_{\mathrm{g}}$ electron is excited from a $\mathrm{Mn}^{3+}$ (bridging, marked A) site to a neighbouring $\mathrm{Mn}^{4+}$ (corner, marked B) site on the same zigzag chain. The green box highlights that the electron now residing on the corner site is experiencing effective intersite Coulomb interaction $V$ with the $e_{g}$ electrons on the other three $\mathrm{Mn}^{3+}$ nearest neighbours. (b) The $\mathrm{e}_{\mathrm{g}}$ energy levels of the $\mathrm{Mn}$ ions. They are degenerate on the $\mathrm{Mn}^{4+}$ site and split by $2 \mathrm{E}_{\mathrm{T}}$ on the $\mathrm{Mn}^{3+}$ site due to Jahn-Teller distortion and adjusted by $\mathrm{E}_{\mathrm{BM}}$ due to the breathing-mode lattice distortion. Only the lower level of the $\mathrm{Mn}^{3+}$ site is occupied by an electron (dark red ball). In the intrasite $\mathrm{d}$ - $d$ transition (the yellow arrow), the $\mathrm{e}_{\mathrm{g}}$ electron is excited to the higher level of the $\mathrm{Mn}^{3+}$ site, requiring the photon energy of $2_{\mathrm{EJT}}$. (c) The intersite $d-d$ transition requires the photon energy of $\mathrm{E}_{J T}+\mathrm{E}_{B M}+3 \mathrm{~V}$. $(\mathbf{d})$ Intersite $z^{2}-z^{2}$ transition, after which the oxygen atoms in between $\mathrm{O}_{\mathrm{l}}$ experience a strong force (open arrow). (e) Meanwhile, the $\mathrm{e}_{\mathrm{g}}$ electron pumped onto the $\mathrm{Mn}^{4+}$ site will relax to a hybrid $\mathrm{z}^{2}$ and $\mathrm{x}^{2}-\mathrm{y}^{2}$ orbital to fit the new oxygen surrounding. When this electronic relaxation is fast, $\mathrm{O}_{1}$ experiences a weaker force from the hybrid orbital, leading to the normal (single time constant) behaviour (right panel). (f) When the electronic relaxation is slow, $\mathrm{O}_{1}$ first experiences a strong force from the $\mathrm{z}^{2}$ orbital and then a weaker force from the relaxed hybrid orbital, leading to the overshoot (two time constants) behaviour (right panel). The present experimental results suggest that the force on $\mathrm{O}_{\mathrm{I}}$ is transmitted onto the $\mathrm{La} / \mathrm{Sr}$ atom in a Newton's cradle-like effect. 
the $\mathrm{Mn}^{3+}$ site (marked A in Figure 4a) into a lower locally occupied $3 z^{2}-r^{2}$ (or $z^{2}$ for shorthand notation) and higher unoccupied $x^{2}-y^{2}$ levels ${ }^{33}$ (see Figure 4b). Recent first-principles electronic structure study of a half-doped manganite ${ }^{15}$ showed that the Jahn-Teller energy gain on the $\mathrm{Mn}^{3+}$ site is $E_{\mathrm{T}}=0.226 \mathrm{eV}$, the breathing mode energy gain on a pair of $\mathrm{Mn}^{3+}$ and $\mathrm{Mn}^{4+}$ sites is $E_{\mathrm{BM}}=0.084 \mathrm{eV}$ and the effective intersite Coulomb repulsion is $V_{\text {eff }}=0.44 \mathrm{eV}$. On the basis of these theoretical results, as shown in Figure $4 \mathrm{~b}$, an on$\mathrm{Mn}^{3+}$-site $d-d$ transition costs an energy of $\sim 2 E_{\text {JT }}=0.46 \mathrm{eV}$. On the other hand, as shown in Figure $4 \mathrm{a}$ and $\mathrm{c}$ for an intersite $d-d$ transition, the energy cost is $\sim 3 V_{\text {eff }}+E_{\mathrm{JT}}+E_{\mathrm{BM}}=1.63 \mathrm{eV}$. The band dispersion will modify the threshold for the photoinduced intrasite and intersite $d$ - $d$ transitions by about \pm 0.25 and $\pm 0.45 \mathrm{eV}$, respectively. A value of $1.55 \mathrm{eV}$ falls into the excitation energy range for the intersite $d-d$ transition only $(1.63 \pm 0.45 \mathrm{eV})$ and it is close to the $1.63 \mathrm{eV}$ from the centre of band mass consideration, which implies high transition rates. Hence, an intersite $d-d$ transition is much more likely to be induced by the $1.55 \mathrm{eV}$ optical photons than the other transitions. To be more specific, the photoexcitation causes an intersite $z^{2}-z^{2}$ transition because the matrix element between neighbouring $z^{2}$ and $x^{2}-y^{2}$ orbitals is vanishing by symmetry (Figure $4 d$ ). The initial $z^{2}$ state of the electron pumped on the $\mathrm{Mn}^{4+}$ site will relax into a hybrid $z^{2}$ plus $x^{2}-y^{2}$ orbital to fit the new non-Jahn-Teller-distorted oxygen surroundings. Depending on the speed of this electronic relaxation, as illustrated in Figure $4 \mathrm{e}$ and $\mathrm{f}$, the La/Sr overshoot behaviour may (for slow relaxation) or may not (for fast relaxation) be exhibited, when the electron relaxation is slow or, relatively speaking, the lattice response is fast, the lattice can display two distinct behaviours corresponding to the two different electronic states before and after the relaxation. In strong contrast, the $\mathrm{Mn}^{4+}$ displacement is dragged by the on-site electronic relaxation, leading to its normal behaviour. After the electronic relaxation is complete, the Jahn-Teller $\mathrm{O}$ and $\mathrm{Mn}^{4+}$, and $\mathrm{La} / \mathrm{Sr}$ lattice distortions, can converge.

\section{Summary}

Our experimental results and theoretical analyses consistently reveal that the relaxation of the electron pumped away from the $\mathrm{Mn}^{3+}$ site in $\mathrm{LaSr}_{2} \mathrm{Mn}_{2} \mathrm{O}_{7}$ is slow and can be caught up by even La and $\mathrm{Sr}$, the heaviest elements in the system. This is direct evidence for polaron formation, and shows that the motion of electrons within the cloth of atomic lattice distortions dominates the behaviour of this system, and thus is likely to be the key to the understanding of the doped manganites. In addition to the intriguing coupled phenomenologies of $\mathrm{OO}, \mathrm{CO}$ and colossal magnetoresistance in the manganites, many exotic physical properties emerge in materials based on transition metal elements. ${ }^{8} \quad$ Strong electron-electron interactions on the transition-metal ions themselves are known to be a key driving force in determining those properties; however, the critical relevance of the lattice degrees of freedom is often a highly debated issue. Intrinsically strong electron-electron and electronlattice interactions can in principle slow down the electrons and favour the formation of polarons. By using the manganite $\mathrm{LaSr}_{2} \mathrm{Mn}_{2} \mathrm{O}_{7}$ as a test bed, we have demonstrated here the capability of MeV-UED to quantify very short timescale correlations of the atomic and electronic systems in a complex material. Its strength is that it can simultaneously measure a large number of reflections and thus provide time-dependent quantitative analysis of the atom-specific lattice dynamics on the picosecond and subpicosecond timescale. We anticipate wide application of the technique to correlated materials in general and the cuprate superconductors in particular, where, for example, transient superconductivity approaching room temperature was reported to be induced by pump photons close in energy to a specific lattice oscillation mode. ${ }^{4,9,10}$

\section{METHODS}

Sample and experimental set-up

$\mathrm{LaSr}_{2} \mathrm{Mn}_{2} \mathrm{O}_{7}$ single crystals were grown in a floating zone furnace. The crystal sample was cut along the layer-stacking direction and thinned to $80 \mathrm{~nm}$ thickness with mechanical polishing and low-energy $\mathrm{Ar}^{+}$ion-milling to allow electron transmission and ensure that the whole probed volume is properly pumped by optical pulses with the penetration depth of $\sim 120 \mathrm{~nm}$. Then, the flake was transferred to a Cu grid and characterised at room temperature and $77 \mathrm{~K}$ using a $300 \mathrm{keV}$ field-emission transmission electron microscope. In the MeV-UED experiments, optical pulses with duration of $100 \mathrm{fs}$ and a centre wavelength of $800 \mathrm{~nm}(1.55 \mathrm{eV})$ were focused down to $1.5 \mathrm{~mm}$ on the sample to trigger electronic excitations and crystal structure evolution. At a specific time delay, well-synchronised 2.8 MeV electron pulses with the time resolution of $130 \mathrm{fs}$ were collimated to $200 \mu \mathrm{m}$ in the pumped area. Nearly 10,000 electron diffraction patterns were recorded for various pump-probe time delays, sample temperatures and pump fluences. The sample was not damaged by the high-energy electron pulses as the experimental results were highly reproducible.

The high-quality electron beams $\left(10^{6}\right.$ electrons per bunch, of length $100 \mathrm{fs}$, with a longitudinal and transverse coherence length of $\sim 2$ and $\sim 10 \mathrm{~nm}$, respectively) were produced by using a unique Brookhaven National Laboratory-type photocathode radio frequency gun with a deflecting cavity. ${ }^{30,31}$ The ultrahigh electron energy significantly minimises space-charge effects, allowing for a high flux of electrons in extremely short pulses. MeV electrons can also penetrate thicker samples and significantly reduce multiple scattering effects in favour of quantitative analyses due to their longer mean free path than $\sim 50 \mathrm{keV}$ electrons typically used in DC-UED. Moreover, as electrons interact with matter more strongly than X-rays, ${ }^{34,35}$ the pump-probe approach with electrons yields a large number of elastic scattering events and enables observations that are sensitive to electrons and atomic motions.

\section{Diffraction analysis}

For time-resolved crystal structure refinement, the Bloch wave method, a well-established quantitative dynamical diffraction approach in which the multiple scattering effects are taken into account, was used to calculate the electron diffraction pattern of the crystal for the various lattice distortion modes considered in this paper. ${ }^{36}$ The results were compared with the experimental $\mathrm{OO}, \mathrm{CO}$ and Bragg diffraction intensities to determine the roles of various lattice distortion modes at each pumpprobe time delay. In addition, the sample geometry $(80 \mathrm{~nm}$ in thickness with a $0.6^{\circ}$ bending angle along the [040] direction) was determined by matching the intensities of $\sim 40$ Bragg and $\mathrm{OO}$ spots in the (001) zone before time zero using the atomic positions based on neutron diffraction ${ }^{14}$ and refined by electron diffraction experiments.

\section{ACKNOWLEDGEMENTS}

This work was mainly supported by the Materials Science and Engineering Divisions, Office of Basic Energy Sciences of the U.S. Department of Energy under Contract No. DESC0012704 and BNL Laboratory Directed Research and Development (LDRD) funds 2010-010 and 2012-22. The work at Postech was supported by the Max Planck POSTECH/KOREA Research Initiative Program (Grant No. 2011-0031558) through NRF of Korea funded by MSIP. The work at Rutgers University was supported by the DOE under Grant No. DOE: DE-FG02-07ER46382.

\section{CONTRIBUTIONS}

$\mathrm{YZ}, \mathrm{JPH}$ and XW conceived the project; $\mathrm{JL}, \mathrm{PZ}$ and TK carried out the experiment; JL and LW analysed the data with assistance of JT and WY helped interpretation. YZ, JL and WY wrote the manuscript and RJC revised the manuscript; LSMO single crystal was synthesised by JY with guidance from S-WC, FC and JM involved in discussions on the underlying physics. All the authors contributed to the manuscript.

\section{COMPETING INTERESTS}

The authors declare no conflict of interest.

\section{REFERENCES}

1. Fröhlich, H., Peltzer, H. \& Zienau, S. XX. Properties of slow electrons in polar materials. Phil. Mag. 41, 221-242 (1950). 
2. Cho, A. Superconductivity's smorgasbord of insights: a movable feast. Science 332, 190-192 (2011).

3. Millis, A. Lattice effects in magnetoresistive manganese perovskites. Nature $\mathbf{3 9 2}$ 147-150 (1998)

4. Fausti, D. et al. Light-induced superconductivity in a stripe-ordered cuprate. Science 331, 189-191 (2011).

5. Anderson, P. W. Is there glue in Cuprate superconductors? Science 316, 1705-1707 (2007).

6. Newns, D. M. \& Tsuei, C. C. Fluctuating Cu-O-Cu bond model of high-temperature superconductivity. Nat. Phys. 3, 184-191 (2007).

7. Yin, W. G., Volja, D. \& Ku, W. Orbital ordering in $\mathrm{LaMnO}_{3}$ : electron-electron versus electron-lattice interactions. Phys. Rev. Lett. 96, 116405 (2006).

8. Dagotto, E. Complexity in strongly correlated electronic systems. Science $\mathbf{3 0 9}$ 257-262 (2005).

9. $\mathrm{Hu}, \mathrm{W}$. et al. Optically enhanced coherent transport in $\mathrm{YBa}_{2} \mathrm{Cu}_{3} \mathrm{O}_{6.5}$ by ultrafast redistribution of interlayer coupling. Nat. Mater. 13, 705-711 (2014).

10. Mankowsky, R. et al. Nonlinear lattice dynamics as a basis for enhanced superconductivity in $\mathrm{YBa}_{2} \mathrm{Cu}_{3} \mathrm{O}_{6.5}$. Nature 516, 71-73 (2014).

11. Li, T. et al. Femtosecond switching of magnetism via strongly correlated spin-charge quantum excitations. Nature 496, 69-73 (2013).

12. Goodenough, J. B. Theory of the role of covalence in the perovskite-type manganites [La, M (II)] $\mathrm{MnO}_{3}$. Phys. Rev. 100, 564-573 (1955).

13. Li, J. Q., Matsui, Y., Kimura, T. \& Tokura, Y. Structural properties and chargeordering transition in $\mathrm{LaSr}_{2} \mathrm{Mn}_{2} \mathrm{O}_{7}$. Phys. Rev. B 57, 3205-3208 (1998).

14. Argyriou, D., Bordallo, H. \& Campbell, B. Charge ordering and phase competition in the layered perovskite $\mathrm{LaSr}_{2} \mathrm{Mn}_{2} \mathrm{O}_{7}$. Phys. Rev. B 61, 15269-15276 (2000).

15. Volja, D., Yin, W.-G. \& Ku, W. Charge ordering in half-doped manganites: weak charge disproportion and leading mechanisms. Europhys. Lett. 89, 27008 (2010).

16. Allen, P. B. \& Perebeinos, V. Anti-Jahn-Teller polaron in $\mathrm{LaMnO}_{3}$. Phys. Rev. B 60 , 10747-10753 (1999).

17. Hotta, T., Malvezzi, A. \& Dagotto, E. Charge-orbital ordering and phase separation in the two-orbital model for manganites: roles of Jahn-Teller phononic and Coulombic interactions. Phys. Rev. B 62, 9432-9452 (2000).

18. Millis, A., Littlewood, P. \& Shraiman, B. Double exchange alone does not explain the resistivity of $\mathrm{La}_{1-x} \mathrm{Sr}_{x} \mathrm{MnO}_{3}$. Phys. Rev. Lett. 74, 5144-5147 (1995).

19. Jaime, M. et al. Hall-effect sign anomaly and small-polaron conduction in $\left(\mathrm{La}_{1-\mathrm{x}} \mathrm{Gd}_{\mathrm{x}}\right)_{0.67} \mathrm{Ca}_{0.33} \mathrm{MnO}_{3}$. Phy. Rev. Lett. 78, 951-954 (1997).

20. Şen, C., Alvarez, G. \& Dagotto, E. Competing ferromagnetic and charge-ordered states in models for manganites: the origin of the colossal magnetoresistance effect. Phys. Rev. Lett. 98, 127202 (2007).

21. Jooss, C. et al. Polaron melting and ordering as key mechanisms for colossal resistance effects in manganites. Proc. Natl Acad. Sci. UsA 104, 13597-13602 (2007).
22. Wu, L., Klie, R. F., Zhu, Y. \& Jooss, C. Experimental confirmation of Zener-polarontype charge and orbital ordering in $\operatorname{Pr}_{1-x} \mathrm{Ca}_{x} \mathrm{MnO}_{3}$. Phys. Rev. B 76, 174210 (2007).

23. Ehrke, $\mathrm{H}$. et al. Photoinduced melting of antiferromagnetic order in $\mathrm{La}_{0.5} \mathrm{Sr}_{1.5} \mathrm{MnO}_{4}$ measured using ultrafast resonant soft X-Ray diffraction. Phys. Rev. Lett. 106, 217401 (2011).

24. Först, $M$. et al. Driving magnetic order in a manganite by ultrafast lattice excitation. Phys. Rev. B 84, 241104(R) (2011).

25. Beaud, P. et al. A time-dependent order parameter for ultrafast photoinduced phase transitions. Nat. Mater. 13, 923-927 (2014).

26. Eichberger, M. et al. Snapshots of cooperative atomic motions in the optical suppression of charge density waves. Nature 468, 799-802 (2010).

27. Williamson, J. C., Cao, J., Ihee, H., Frey, H. \& Zewail, A. H. Clocking transient chemical changes by ultrafast electron diffraction. Nature 386, 159-162 (1997).

28. Nie, S., Wang, X., Park, H., Clinite, R. \& Cao, J. Measurement of the electronic Gruneisen constant using femtosecond electron diffraction. Phys. Rev. Lett. 96 025901 (2006).

29. Siwick, B. J., Dwyer, J. R., Jordan, R. E. \& Miller, R. J. D. An atomic-level view of melting using femtosecond electron diffraction. Science 302, 1382-1385 (2003).

30. Zhu, P. et al. Femtosecond time-resolved MeV electron diffraction. New J. Phys. 17, 063004 (2015).

31. Zhu, P. et al. Dynamic separation of electron excitation and lattice heating during the photoinduced melting of the periodic lattice distortion in $2 \mathrm{H}-\mathrm{TaSe}_{2}$ Appl. Phys. Lett. 103, 071914 (2013).

32. Wilkins, S. B. et al. Separating the causes of orbital ordering in $\mathrm{LaSr}_{2} \mathrm{Mn}_{2} \mathrm{O}_{7}$ using resonant soft x-ray diffraction. J. Phys. Condens. Matter 18, L323-L329 (2006).

33. Goodenough, J. B. Electronic and ionic transport properties and other physical aspects of perovskites. Rep. Prog. Phys. 67, 1915-1993 (2004).

34. $\mathrm{Ma}, \mathrm{C}$. et al. Strong coupling of the iron-quadrupole and anion-dipole polarizations in $\mathrm{Ba}\left(\mathrm{Fe}_{1-x} \mathrm{Co}_{x}\right)_{2} \mathrm{As}_{2}$. Phys. Rev. Lett. 112, 077001 (2014).

35. Zhu, Y. \& Dürr, H. The future of electron microscopy. Phys. Today 68, 32-38 (2015).

36. Spence, J. C. H. \& Zuo, J. M. Electron Microdiffraction. Plenum Press (1992).

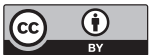

This work is licensed under a Creative Commons Attribution 4.0 nternational License. The images or other third party material in this article are included in the article's Creative Commons license, unless indicated otherwise in the credit line; if the material is not included under the Creative Commons license, users will need to obtain permission from the license holder to reproduce the material. To view a copy of this license, visit http://creativecommons.org/licenses/ by/4.0/

(c) The Author(s) 2016

Supplementary Information accompanies the paper on the npj Quantum Materials website (http://www.nature.com/npjquantmats) 